\title{
APPROCCI COMPUTERIZZATI PER L'INSEGNAMENTO DELLA TRADUZIONE DI LINGUAGGI SPECIALISTICI
}

\author{
Neva Čebron, Università del Litorale, neva.cebron@fhs.upr.si \\ Jadranka Cergol, Università del Litorale, jadranka.cergol@fhs.upr.si
}

\subsection{2/fll.30.2020.13 UDK 004:[371.3:82.03}

L'articolo propone la discussione di un corso sperimentale e innovativo per gli studenti di secondo livello in italianistica insegnando nuovi approcci alla traduzione di testi specialistici. Prendendo in considerazione i principi, i processi e gli approcci proposti da numerosi linguisti (ad es. Wills; Hause; Erdman et al.), il corso ha inizialmente introdotto gli studenti all'analisi testuale prima della traduzione proseguendo con l'esplorazione delle possibilità offerte dalle nuove tecnologie come l'utilizzo dei corpora e degli strumenti di traduzione assistita (CAT), proposti da un certo numero di linguisti dei corpora (ad es. Laviosa; Gavioli; Boulton; Zanettin e Fantinuoli). Gli studenti sono stati introdotti all'uso degli strumenti CAT con il compito di tradurre un documento ufficiale dallo sloveno all'italiano e sono stati in seguito guidati attraverso tutte le fasi di preparazione ed esecuzione della traduzione. I partecipanti dovevano infatti inizialmente analizzare il testo in considerazione sull'acquisizione dei titoli accademici, dopodiché sono state presentate le varie opzioni fornite da dispositivi IT come dizionari online, glossari, database terminologici e corpora linguistici che possono aiutare a esplorare le principali risorse lessicali e problemi di collocazione. Al fine di raffinare ulteriormente le scelte lessicali ed esaminare la principale terminologia specialistica, sono stati introdotti i principi di compilazione di corpora comparativi su piccola scala insieme agli strumenti software per l'esplorazione dei corpora di tale dimensione. I risultati di tale ricerca scientifica sono stati confrontati con le soluzioni di traduzione proposte dai dispositivi di traduzione automatica, nonché con le traduzioni proposte da traduttori professionisti e madrelingua italiani. Le conclusioni tratte dal corso sperimentale hanno implicazioni per le pratiche di insegnamento e soluzioni di traduzione.

Parole chiave: strumenti CAT, corpora di linguaggio comparativo su piccola scala, approcci di insegnamenti della traduzione 


\section{INTRODUZIONE}

Negli ultimi decenni la linguistica dei corpora sta diventando una delle metodologie sempre più ricorrenti nella ricerca della linguistica contrastiva, nonché negli studi della traduzione e terminologia, come comprovato da diversi autori e ricercatori che si sono occupati dell'uso dei corpora nella traduzione e dei benefici che ne hanno tratto traduttori e linguisti (ad es. Boulton; Bernardini; Gavioli; Johansson; Zanettin). II pregio di tale ricerca risulta essere soprattutto l'uso di testi prodotti nella lingua naturale, espressione di comunicazione spontanea tra parlanti nativi in ambiti diversi, poiché $i$ corpora sono raccolte di testi realizzati in una certa lingua in formato elettronico scelti sulla base di criteri specifici (Sinclair 1991; Bowker e Pearson; McEnry et al.). Di conseguenza l'uso dei corpora per $i$ traduttori e studenti di traduzione sembra essere fonte d'informazione linguistica necessaria soprattutto per i testi che rappresentano reali situazioni comunicative; sono inoltre una base indispensabile per gli approcci sviluppati dagli strumenti CAT (Malmkjaer; Riediger).

Numerosi sono quindi anche i ricercatori ed insegnanti che propongono l'uso dei corpora elettronici come strumento didattico sia per l'insegnamento/apprendimento delle lingue straniere, sia per l'analisi linguistica intrinseca alla traduzione e alla ricerca della terminologia dei linguaggi specialistici (ad es. Bernardini, Zanettin, Stewart; Fantinuoli e Zanettin; Olhan). Nell'ambito didattico rimangono comunque alquanto rare le proposte di sfruttamento dei corpora che si spingono oltre alle modalità della loro interrogazione di riferimento esistenti (Gallego-Hernandez).

Si propone quindi di adottare approcci di manipolazione dei corpora più complessi, resi possibili da software recenti. Essi semplificano la loro compilazione fai-da-te e consentono interrogazioni complesse, nonché raccolte di banche dati terminologiche, rendendo così il lavoro di studente/traduttore più indipendente ed autonomo nelle sue scelte traduttive, poiché provvisto di banche dati specializzate basate sull'uso della lingua reale. Con i corpora comparativi tale ricerca linguistica della lingua di partenza quanto della lingua d'arrivo può, in un secondo momento, essere sfruttata anche nell'applicazione dei programmi di traduzione assistita (CAT).

II progetto, presentato in questo articolo, espone quindi gli approcci didattici usati nell'introdurre gli studenti alle possibilità offerte dai corpora comparativi in italiano e in sloveno, per identificare delle scelte basate sulla metodologia empirica nella ricerca traduttiva. $\mathrm{Si}$ tratta di un esperimento che nasce nell'ambito della didattica della traduzione basata sull'uso dei corpora e degli strumenti CAT, finalizzata 
alla formazione di traduttori in grado di lavorare in un contesto specialistico, cioè, nel contesto delle microlingue, nel nostro caso, il linguaggio accademico istituzionale.

Nello specifico, l'articolo descrive gli approcci didattici usati nell'introduzione dei corpora linguistici agli studenti del corso di laurea di secondo livello in italianistica della Facoltà di studi umanistici dell'Università del Litorale nell'ambito di due corsi: Traduzione assistita e Mediazione interculturale delle lingue professionali. Inoltre l'articolo riporta gli approcci addottati dagli studenti nella ricerca delle strutture lessico-grammaticali paradigmatiche e della terminologia disciplinare con l'intento di impiegare questi nuovi dati nell'uso degli strumenti della traduzione assistita.

Alla conclusione dei corsi è stato chiesto agli studenti di presentare i risultati della loro ricerca linguistica e di fare una valutazione dell'esperimento e dell'utilità dell'uso degli corpora linguistici sia nella traduzione specialistica, sia nella formazione professionale di traduttori. Vengono riportate le risposte ottenute dagli studenti in questo merito. In conclusione, proponiamo anche degli sbocchi/suggerimenti per ottimizzare l'approccio didattico e per facilitare l'adozione dell'uso dei corpora dagli studenti anche nel loro futuro lavoro professionale.

\section{PARTE TEORICA: CORPORA, TRADUZIONE, RICERCA}

Negli ultimi decenni l'impiego dei corpora è una pratica metodologica diffusa negli studi sulla traduzione e si basa sull'idea della lingua come fenomeno sociale che, in quanto tale, deve essere investigata a partire da dati reali, forniti da ampie compilazioni di testi originali. La prima studiosa ad intuire l'importanza di tali risorse anche per la formazione di futuri traduttori era Mona Baker (1993) che nella sua ricerca dei corpora paralleli scoprì divergenze paradigmatiche ricorrenti tra l'uso della lingua naturale e la lingua dei testi tradotti (translationese). Auspicò quindi l'applicazione di metodi e mezzi della linguistica computazionale negli studi descrittivi sulla traduzione quanto anche nella didattica della traduzione. Promosse così le idee dei teorici di traduzione (Toury; Vermeer; McEnery e Wilson) che collocano il testo tradotto in un contesto socio-storico-culturale diverso dal testo originale. La traduzione viene quindi percepita come un processo costruttivo e non derivativo, che comporta l'adattamento del testo di partenza, in ottemperanza a norme storico-culturali e disciplinari dell'audience di lettori del testo di arrivo. L'equivalenza non riguarda più soltanto le "norme della traduzione" prescrittive, ma viene descritta 
e determinata dal contesto socio-culturale di ricezione (Laviosa; Olohan 2004).

Tali nuovi concetti vengono promossi in base alle analisi dei corpora linguistici, inizialmente in base a dati ottenuti dai corpora paralleli bilingui (testi nella lingua di partenza contrastati a testi nella lingua di arrivo), ed in seguito anche dai corpora paragonabili monolingui (testi originali nella lingua $A$ contrastati a testi tradotti nella lingua $A$, simili per forma e contenuto) e dai corpora comparabili in entrambe le lingue contrastate (testi originali nella lingua A contrastati a testi originali nella lingua $B$, simili per forma e contenuto). In tal modo vengono studiate diverse dimensioni del testo come la densità e la varietà lessicale (Laviosa), l'uso di sequenze (semi)grammaticali (Olohan e Baker), le collocazioni e le stringhe di parole (TirkkonenCondit; Mauranen).

Da queste ricerche si è constatato che i corpora comparativi sono particolarmente adatti per lo studio comparativo della strutturazione paradigmatica e sequenziale del lessico (collocazioni) e della terminologia. Mettendo a confronto le opzioni paradigmatiche delle parole chiave e le strutture sequenziali in due lingue si mettono a fuoco le loro realizzazioni linguistiche, il che ci aiuta ad intravedere le opzioni sviluppate in ogni singola lingua come strutture comparabili e paradigmi di corrispondenze facilmente esplorabili (Ashton 2000; Olohan 2004; Baker 2009; Prandi). Secondo gli studi di lessicologia e terminologia contrastiva (Kenny e Way; Sinclair 2007; Soffritti) tale ricerca offre al traduttore la possibilità di individuare una gamma di realizzazioni linguistiche a disposizione nelle singole lingue e di usare nella traduzione dei repertoremi (collocazioni e locuzioni esistenti nella lingua d'arrivo) invece dei textemi (formulazioni creative dell'uso nella lingua di partenza) (Toury). Le analisi dei corpora comparativi ci permettono inoltre di inferire possibili strutture sequenziali, ovvero schemi costanti di svolgimento dell'evento linguistico, quanto anche di verificare opzioni paradigmatiche a livello linguistico e costanti che sottostanno a particolari classi di eventi linguistici (Berruto) nella lingua di arrivo. Tali forme stereotipiche di mezzi linguistici (di tipo lessicale, morfologico, fraseologico e sintattico) caratterizzano in maniera specifica alcuni generi testuali propri di aree tematiche settoriali (De Mauro) e rispondono alle esigenze di comprensione ottimale a livello specialistico, in quanto esse richiedono precisione, univocità e concisione anche nella loro traduzione in altre lingue.

Il punto di partenza in tutte queste analisi e ricerche linguistiche è il concetto di locuzione o collocazione, ovvero la distribuzione delle co-occorrenze delle varie unità lessicali (Sinclair et 
al.; Louw; Stubbs; Hoey). Attraverso l'individuazione delle parole chiave, l'interrogazione dei corpora paralleli o comparativi, il text mining, ci permette di intravedere l'intreccio del lessico e della grammatica in lingue diverse, data la natura fraseologica della lingua, ed inoltre di decidere se adottare una strategia di mantenimento delle strutture e lo stile della lingua di partenza, oppure una strategia volta a rendere la traduzione più vicina alla lingua e cultura di arrivo (Bernardini).

Ulteriori analisi di dati e strutture estrapolati dai corpora comparativi ci permettono di stabilire corrispondenze lessicali e terminologiche, nonché la categorizzazione e classificazione dei dati estratti e il loro riversamento sotto forma di database alla base dei strumenti CAT (Riediger).

\section{PARTE APPLICATIVA: IL CORSO SPERIMENTALE:} METODOLOGIA E APPROCCI

Il corso di laurea di secondo livello in italianistica della Facoltà di studi umanistici dell'Università del Litorale offre agli studenti anche un corso di traduzione di testi specialistici e un corso di traduzione assistita. All'interno di questi due corsi è stato introdotto nell'anno accademico 2018/2019 un nuovo approccio alla traduzione di questo tipo di testi, utilizzando le possibilità offerte dalle nuove tecnologie come i corpora elettronici e gli strumenti di traduzione assistita (CAT computer assisted translation). Per dimostrare la validità del metodo scelto si è deciso di tradurre dallo sloveno all'italiano un tipo di testo da un linguaggio amministrativo-accademico, più precisamente due documenti ufficiali, cioè il Regolamento per la stesura e la discussione della tesi di dottorato e i Criteri e parametri per l'acquisizione dei titoli accademici.

\section{1 II progetto: i partecipanti al corso di traduzione}

Entrambi i corsi di traduzione si sono svolti nel secondo semestre dell'anno accademico 2018/2019, ed erano indirizzati ad un piccolo gruppo di quattro studenti del corso di Laurea magistrale in Italianistica. Gli studenti avevano una conoscenza della lingua italiana molto avanzata (QCER C2), una buona competenza tecnica, ma scarsa esperienza di uso di strumenti informatici per la traduzione. II gruppo era composto da studenti laureati in Italianistica presso la Facoltà di studi umanistici e iscritti al primo anno del secondo livello di Italianistica, indirizzo traduttologico. Già durante lo studio al primo livello avevano ricevuto alcune nozioni base di micro-lingue, della sociolinguistica dell'italiano e dei principali strumenti di traduzione. Gli 
studenti hanno accettato con entusiasmo il compito di lavorare insieme sul testo in questione.

La realizzazione dei corsi prevedeva 30 ore di lezioni e attività di laboratorio per ogni modulo (il corso di mediazione interculturale di testi specialistici e il corso di traduzione assistita). Alla fine dei due moduli era richiesto che gli studenti presentassero la traduzione di uno dei testi proposti e una relazione finale che discuteva i pregi e i difetti dell'analisi linguistica basata sui corpora linguistici, con esempi di comparazione terminologica contrastiva collocati nell'ambito del linguaggio accademico istituzionale.

\section{2 II progetto: La metodologia}

Dopo un'introduzione teorica dei principi, processi e approcci proposti da numerosi linguisti (Cortellazzo e Pellegrino; Serianni; Bruni e Raso; Nigro; Balboni), il corso di traduzione di testi specialistici ha inizialmente introdotto gli studenti all'analisi testuale che precede la traduzione del testo.

II programma di studio del modulo sulla traduzione assistita si basa invece su una vasta e diversificata area di traduttologia che oggi si fonda sull'esplorazione dei corpora, più precisamente, sul ramo di queste ricerche, essenzialmente pedagogiche, in cui i corpora vengono utilizzati come risorse didattiche per la formazione dei futuri traduttori e per approfondire l'apprendimento linguistico (ad es. Aston 2001; Bernardini et al.).

Lo scopo principale del corso era di

- introdurre gli studenti ai principali concetti teorici ed applicativi della linguistica dei corpora

- presentare l'uso applicativo di un numero limitato di strumenti elettronici

- indicare i principi base della compilazione di un corpus linguistico

- concedere di acquisire dimestichezza con l'analisi linguistica basata sui corpora comparativi

- introdurre gli approcci all'estrazione della terminologia disciplinare comparata dai corpora specialistici

- presentare l'uso della terminologia come banche terminologiche per gli strumenti CAT.

L'approccio adottato ha introdotto gradualmente gli studenti alle nuove strategie della traduzione assistita e all'uso degli strumenti elettronici per evitare di sommergerle di informazioni e dati e per permettere loro di acquisire padronanza delle varie strategie di consultazione dei corpora e scoprire progressivamente i vantaggi 
dell'analisi linguistica dei corpora sia per l'apprendimento e perfezionamento della lingua, sia per la comprensione e l'analisi dei linguaggi specialistici. In tutte le fasi del corso sperimentale l'apprendimento si è svolto in base ad attività che riportavano al compito di tradurre $i$ due documenti ufficiali in un linguaggio amministrativo-accademico dallo sloveno all'italiano.

Inizialmente è risultato necessario spiegare agli studenti perché i traduttori professionisti si avvalgono della consultazione diretta dei corpora creati ad hoc come fonte di informazioni sulla terminologia e per le indicazioni sulle strutture lessico-grammaticali e sul registro. Dato che il corso proponeva agli studenti approcci nuovi all'analisi della lingua, bisognava quindi delineare brevemente il quadro teorico delle attività che si sarebbero svolte in classe e introdurre i concetti base della linguistica computazionale (ad es. liste di frequenza, densità lessicale, parole chiave, collocazioni, locuzioni, frasi fatte, clichés, prosodia semantica). Inoltre sono stati forniti agli studenti diversi articoli sulla ricerca della traduzione abbinata allo sfruttamento delle risorse elettroniche, in particolar modo, all'analisi dei corpora comparativi e all'estrazione dei dati lessico-grammaticali nonché terminologici.

La seconda fase del corso sperimentale si è focalizzata sull'uso di varie risorse on-line: dizionari informatizzati monolingui, dizionari informatizzati bilingui basati su memorie di traduzione, glossari elettronici, database terminologici, corpora monolingui in sloveno ed italiano (elencati nella Tabella 2 in Appendice). I discenti dovevano inizialmente analizzare i documenti presi in considerazione con varie risorse come dizionari online, glossari e interrogazioni di Internet, che possono aiutare a esplorare le principali risorse lessicali in una lingua. Constatando che sono un'ottima risorsa, ma non bastano per fare un controllo più accurato e scrupoloso sull'uso del termine ricercato, soprattutto perché per alcune combinazioni linguistiche non esistono dizionari per vari settori disciplinari, si è progredito all'analisi linguistica dei testi sia tramite i corpora monolingui in sloveno e italiano (GigaFida, Perugia Corpus), sia in base alle corrispondenze traduttive fornite dagli esempi nei corpus bilingui di traduzione in italiano (Linguee, Glosbe, Eurocorpus). Con la ricerca effettuata con questi strumenti si è ottenuto l'elenco di tutte le occorrenze e concordanze di una stringa nei corpora interrogati, mentre la manipolazione delle stringhe di parole ha permesso di identificare collocazioni comuni e locuzioni tipiche in ognuna delle due lingue. Confrontando i dati ricavati dall'analisi dei corpora di riferimento in italiano (compilazioni di documenti su vasta scala, riportando l'uso rappresentativo delle 
diverse varietà scritte e parlate della lingua italiana) con l'informazione lessicale sulle collocazioni frequenti in lingua italiana comune riportata nel Dizionario delle collocazioni (Tiberii 2018), gli studenti hanno scoperto un nuovo approccio all'analisi dell'uso della lingua che hanno in seguito usato nella ricerca lessico-grammaticale per le loro traduzioni.

A questo punto si è avviata la compilazione dei corpora fai-date su piccola scala del settore accademico-amministrativo in sloveno e in italiano. Si è discussa la struttura, la selezione e il bilanciamento dei due corpus comparativi in base ai documenti reperibili sul Web, per poi scaricare i documenti rilevanti creando così dei corpora comparativi di oltre 25.000 parole per ogni lingua in esame (vedi anche la Tabella 3 in Appendice). Nella ricerca contrastiva delle caratteristiche testuali e lessico-grammaticali dei linguaggi settoriali specifici i corpora di almeno 25.000 parole sono considerati adeguati da diversi ricercatori (Hüttner et al.; Ackerley; Chen e Flowerdew).

Una volta costruita la tabella, si è proceduto a trasferire i documenti in due cartelle separate, una per i documenti in lingua italiana e una per i documenti in lingua slovena, da utilizzare per I'analisi con lo strumento AntConc, scaricabile gratuitamente (Anthony), per condurre uno studio testuale approfondito dei corpora creati. Lo strumento permette di avviare l'analisi delle liste di frequenza e delle concordanze dei termini del linguaggio specialistico, chiaramente evidenziando le parole contenute nel corpus a seconda della ricerca avviata. Contrastando i paradigmi lessicali e sintagmatici nell'ambito specialistico in entrambe le lingue, il procedimento ha aiutato a familiarizzare gli studenti con i paradigmi della variazione nelle due lingue, ma anche nell'ambito di variazioni di registro in una lingua.

Constatando l'utilità di tali interrogazioni dei corpora strettamente specializzati si è dovuto pur ammettere le limitazioni dei corpora su piccola scala riguardo i dati linguistici forniti e la difficoltà di individuare il lessico tecnico chiave specifico dei due corpora.

Per risolvere il problema dell'estrazione della terminologia chiave si è deciso di esplorare la piattaforma Sketch Engine, l'uso della quale è gratuito per le istituzioni universitarie. Essa offre anche lo strumento per la compilazione di corpora BootCat. Dopo aver scaricato i documenti dei nostri due corpora specializzati in questa piattaforma il programma ci ha permesso l'analisi terminologica automatica e l'estrazione della terminologia chiave specifica che consiste nel "selezionare le parole da analizzare non in funzione della loro frequenza assoluta, bensì del loro sovra-sotto uso rispetto all'uso 
medio in un linguaggio di riferimento" (Bolasco), nel nostro caso in contrasto al corpus di riferimento Italian Web 2016 (itTenTen16) per I'italiano e in contrasto al corpus Slovenian Web 2015 (sITenTen15) per lo sloveno. Le schede di terminologia chiave in entrambe le lingue hanno permesso un'avanzata ricerca contrastiva e l'identificazione delle collocazioni terminologiche tipiche nonché le lacune linguisticoculturali.

Le informazioni terminologiche estratte in questo modo dai corpora comparativi sono state successivamente utilizzate per compilare le liste di parole chiave in formato Excel, trasferite in forma di banche dati terminologici sulla piattaforma virtuale di traduzione assistita MemSource. II contenuto delle nostre banche dati terminologiche ha permesso al programma di scegliere l'equivalente corretto nella lingua target e in conseguenza di tradurre esattamente il testo di arrivo.

Dopo aver manipolato ed interrogato $i$ documenti dei nostri corpora specialistici con diversi strumenti il programma ha permesso agli studenti di acquisire una buona conoscenza del linguaggio accademico-amministrativo in entrambe le lingue e quindi di costruire il loro corpus mentale che ha facilitato l'identificazione delle buone scelte della traduzione automatica proposte dal programma Memsource, ossia di correggerle con corrispondenze più adeguate, in quanto anche in queste circostanze la scelta finale spetta al traduttore.

\section{3 Discussione}

Con l'aiuto degli strumenti citati è stato costruito un database terminologico contenente i concetti più frequenti e i relativi equivalenti nell'altra lingua, oltre alle definizioni e agli esempi d'utilizzo. Si sottolinea che gli esempi, le collocazioni con aggettivi e i verbi presenti nella Tabella 1 in Appendice sono stati riportati nell'analisi per la loro frequenza d'uso. Ciò significa che gli esempi citati sono emersi dall'analisi di tutti i corpora, ovvero strumenti di cui si è avvalso. Questo procedimento ha reso possibile scoprire quali sono effettivamente le parole più comunemente usate per la definizione di determinati concetti e analizzare il contesto nel quale i termini vengono usati, il che risulta essere più proficuo e utile nella ricerca della scelta linguistica più adeguata.

Dall'analisi è emerso innanzitutto che per ottenere risultati affidabili la banca dati deve essere sostanziosa ovvero contenente un maggior numero di documenti possibili. Questo è stato anche uno dei problemi più difficili, dato che l'ambito dato (ambito accademico linguaggio istituzionale) è un ambito relativamente ristretto: in Slovenia 
esistono solo tre università pubbliche, oltre al fatto che sia in Italia che in Slovenia non sono accessibili on-line tutti i regolamenti di tutte le Facoltà. Dopo un'accurata ricerca on-line sono stati scelti quindi i documenti più pertinenti e inseriti nel corpus. Anche in questo caso però spesso non si è potuti arrivare a delle traduzioni appropriate ossia non si sono trovate corrispondenze nelle due lingue; in base a consultazioni con traduttori di madrelingua italiana si è potuto infatti constatare che per alcuni termini specifici ovvero collocazioni, nell'altra lingua non esiste un corrispondente appropriato. Ad esempio, nelle fasi di ricerca nei corpora disponibili online quali il Perugia Corpus, si è inizialmente pensato di trovare un corrispondente letterale (per program è stato cercato *programma di studi, ma in realtà la corrispondenza più adeguata in italiano è risultata essere piano di studi; si è arrivati alla stessa conclusione anche per il termine ocena, il cui corrispondente non è *voto, ma valutazione). Nonostante l'iniziale difficoltà, il lavoro svolto ha permesso di arrivare a corrispondenze più appropriate nella lingua target anche in merito alle collocazioni (izvolitev $v$ nazive corrisponde a acquisizione del titolo e non a *assegnazione del titolo accademico come inizialmente ipotizzato). Lo stesso problema si è presentato in merito alle denominazioni dei titoli accademici, non solo perché nella lingua target una determinata posizione all'interno del mondo accademico veniva definita in maniera diversa, ma anche perché spesso non esistevano denominazioni neanche approssimative per alcuni titoli accademici (učitelj veščin, visokošolski učitelj...): in Italia infatti il personale tecnicoamministrativo è raggruppato in un'unica categoria, mentre invece le mansioni di questi collaboratori in Slovenia vengono definite più specificatamente. La difficoltà principale è risultata essere quindi nell'enorme differenza tra i sistemi educativi in Italia e in Slovenia, a causa della quale è spesso difficile trovare corrispondenze esatte nelle due lingue per la terminologia specifica. Si tratta, dunque, così come anche per le collocazioni, di espressioni uniche che non possono essere tradotte parola per parola, ma devono essere tradotte idoneamente nel pieno rispetto dei due sistemi universitari differenti che sono stati presi in esame. Nel caso delle collocazioni, le parole richiedono una diversa compagnia in diverse lingue e diverse culture. In questi casi l'unica soluzione possibile risulta quella di trovare un termine che si avvicini quanto possibile al significato nell'altra lingua e usarlo in maniera coerente all'interno del testo.

In classe si è discusso parecchio sulle scelte lessicali ottimali da adottare per determinati termini. A tal proposito, i corpora ci hanno consentito una scelta più ampia di possibilità e hanno posto rimedio $a$ 
questo tipo particolare di traduzione in tre diversi modi: trovando una frase fatta simile, parafrasando o, infine, eseguendo una sorta di sostituzione stilistica. In molti casi abbiamo quindi seguito la linea guida principale, scegliendo prima un corrispettivo adeguato e preparando un glossario da inserire nelle memorie di traduzione e del termbase della stazione di lavoro per il traduttore MemSource che ci ha permesso di usare il termine scelto in modo coerente in tutti i testi.

Dato che è stata la prima volta che un testo di un linguaggio istituzionale dell'ambito accademico è stato tradotto nella combinazione sloveno-italiano, abbiamo dovuto fare delle scelte che potranno diventare normative con l'uso dei termini tradotti in italiano. L'uso della metodologia dei corpora ci ha permesso di avere un'ampia gamma di possibilità e di scegliere il termine più adeguato alla struttura della lingua italiana.

Le soluzioni scelte e il rispettivo confronto con le soluzioni offerte precedentemente dai glossari e dizionari on-line, nonché quelle proposte dalla piattaforma di traduzione assistita di MemSource Cloud, sono elencati nella Tabella 1 in Appendice. Nei casi in cui le soluzioni trovate con l'aiuto dei corpora sono diverse dalla ricerca eseguita con i glossari on-line, i termini sono segnati con l'asterisco $\left({ }^{*}\right)$.

\section{4 Commenti e riscontri degli studenti}

Alla conclusione dei due corsi di Traduzione è stato chiesto agli studenti di presentare i risultati della loro ricerca linguistica in forma di un saggio argomentativo riportando sia i risultati della ricerca dei corpora, sia una valutazione del progetto sperimentale in termini dell'utilità dell'uso dei corpora linguistici nella traduzione specialistica e nella formazione professionale di traduttori. Alcuni risultati della ricerca sono elencati nella Tabella 2 in Appendice.

I commenti sul progetto riportati dagli studenti nei loro saggi fanno intuire un profondo coinvolgimento con i nuovi metodi di ricerca linguistica basata sui corpora. Infatti delineano chiaramente i pregi e i limiti di tale approccio:

I corpora, sicuramente, negli ultimi anni stanno rappresentando un valido strumento di aiuto nell'insegnamento e nell'apprendimento delle lingue. In qualità di raccolte di testi prodotti in maniera autentica da parlanti in contesti comunicativi reali, in tempi recenti hanno subito un notevole sviluppo, pertanto, è possibile disporre sempre più di una mole crescente di dati su usi linguistici reali. (S1)

Dall'analisi dei corpora di dimensioni modeste è emerso innanzitutto che per ottenere risultati affidabili la banca dati deve essere sostanziosa ovvero contenente un maggior numero di documenti possibili. (S2) 
Nonostante l'iniziale difficoltà (di maneggiare $i$ dati riportati dai corpora), l'approccio ci ha permesso di arrivare a corrispondenze più appropriate nella lingua target anche in merito alle collocazioni. (S2)

Prendono in considerazione anche l'utilizzo dei corpora come risorse per la traduzione specializzata:

In campo traduttologico, poi, il corpus viene usato in modo pressoché contrastivo, comparando quanto emerso dall'analisi di due o più corpora. Nel nostro caso, dunque, si è provveduto alla creazione di un corpus comparativo in italiano e in sloveno, in materia di dottorato. (S1) Per quanto riguarda la terminologia, i corpora comparativi hanno dato più credibilità alle relative traduzioni, poiché si tratta di documenti ufficiali autentici, e non di una traduzione istantanea parola per parola. (S2)

Dopo aver svolto questo lavoro gli studenti hanno intravisto l'aspetto sociolinguistico della traduzione:

$\mathrm{Nel}$ nostro percorso di conoscenza dei corpora abbiamo usufruito di diversi strumenti di traduzione assistita, visto che i dizionari specifici spesso non bastano se si intende fare un controllo più approfondito sull'uso di un determinato termine specialistico, soprattutto perché nei dizionari/vocabolari non esistono alcune delle combinazioni linguistiche di nostro interesse. (S2)

La terminologia, inoltre, è fortemente legata alle due diverse culture, quella italiana e quella slovena, che, non solo non usa sempre $i$ corrispettivi relativi disponibili nei comuni dizionari bilingui, ma si adatta ai due diversi sistemi sociali presenti nei due stati. Si tratta, dunque, così come anche per le collocazioni, di espressioni uniche che non possono essere tradotte parola per parola, ma devono essere tradotte idoneamente nel pieno rispetto dei due sistemi universitari differenti che sono stati presi in esame. (S1)

Dalle discussioni nel Focus Group tenute alla fine del corso è emerso che all'inizio dei lavori le studentesse hanno riscontrato qualche difficoltà ad orientarsi nell'uso dei corpora a causa delle molteplici possibilità che vengono offerte. Man mano però che durante le lezioni abbiamo imparato a scegliere le opzioni da utilizzare hanno cominciato ad apprezzare il metodo che offre molteplici possibilità di traduzione. È stato molto apprezzato il fatto che la terminologia e le collocazioni venivano ricercate su testi autentici il che ha permesso una maggiore comprensione del funzionamento di entrambe le lingue. Alla conclusione del corso le studentesse hanno infatti ammesso di aver individuato parecchie strutture linguistiche dell'italiano alle quali prima non avevano neanche pensato o non ci avevano fatto caso durante gli anni di studio della lingua italiana. 


\section{Conclusione}

In conclusione è possibile asserire che i risultati emersi dall'uso dei corpora hanno dimostrato che si tratta di uno strumento valido e utile da utilizzare anche durante i corsi di studio in traduzione; è uno strumento che permette infatti di trovare informazioni specifiche difficilmente reperibili in un semplice vocabolario/dizionario. L'uso di tale metodologia risulta essere soprattutto utile e proficuo per un gruppo di studenti che hanno già un'elevata conoscenza della lingua e/o per studenti che hanno una delle due lingue come lingua madre. Confrontare infatti le strutture sintattiche di diverse lingue ha aiutato a focalizzare meglio la struttura delle singole parti del discorso e comprendere alcune dinamiche linguistiche che sono intrinseche nella propria lingua madre. Questo tipo di approccio linguistico è inoltre molto utile proprio per il fatto che viene svolto su testi reali e quindi utilizzati in un possibile ambito lavorativo per gli studenti che hanno cosi a disposizione esempi pratici da poter usare anche nella propria carriera professionale. II lavoro di traduzione diventa così un esercizio ideale per scoprire, conoscere e approfondire anche la propria lingua madre, indistintamente dal fatto se lo studente intende continuare sulla via professionale della traduzione o decidere per uno studio linguistico più ampio. Così come è stato spiegato dalle studentesse, infatti, gli esercizi di traduzione rappresentano un'importante ambito di confronto tra lingue e culture diverse ed è perciò auspicabile inserirle sistematicamente nello studio delle lingue in contatto. L'uso dei corpora permette questo tipo di esercizi proprio per il pregio di usare testi autentici dal mondo lavorativo. È doveroso comunque sottolineare che si tratta di uno strumento ancora poco utilizzato nell'ambiente accademico perché presenta alcune difficoltà di utilizzo nella prima fase di familiarizzazione. Infatti questo tipo di strumenti inizialmente appare di difficile consultazione a causa delle moltissime possibilità di analisi che offre all'utente. Una volta capito però il meccanismo di funzionamento risultano molto utili non solo per la traduzione di testi e analisi dei vocaboli ma anche per capire in generale l'uso delle lingue. Si propone quindi l'introduzione di questo strumento durante le ore di lezioni per permettere agli studenti di familiarizzare con essi già nel corso degli anni di studio.

\section{Bibliografia:}

Ackerley, Katherine. "Effects of corpus-based instruction on phraseology in learner English." Language Learning and 
Technology 21.3 (2017): 195-216. Http://lt.msu.edu/issues/october2017/ackerley.pdf. 15 Dec 2018.

Anthony, Laurence. "AntConc (Version 3.5.8m Windows) [Computer Software]." Tokyo, Japan: Waseda University. Http://www.laurenceanthony. net/>. 2 Jan 2019.

Aston, Guy. "I corpora come risorsa per la traduzione e l'apprendimento." I corpora nella didattica della traduzione. Eds. Silvia Bernardini e Federico Zanettin. Bologna: CLUEB, 2000. 21-29.

Aston, Guy (ed.). Learning with corpora. Bologna: CLEUB; Houston TX: Athelstan, 2001.

Baker, Mona. "Corpus Linguistics and Translation Studies: Implications and Applications." Text and Technology: In honour of John Sinclair. Eds. Mona Baker, Gill Francis ed Elio Tognini-Bonelli. Amsterdam: John Benjamins Publishing Company, 1993. 233250.

A corpus-based view of similarity and difference in translation.

Amsterdam: John Benjamins Publishing Company, 2004.

Balboni, Paolo. Le microlingue scientifico professionali. Torino: Utet libreria, 2002.

-------La comunicazione interculturale. Venezia: Marsilio, 2007.

Baker, Paul. Contemporary Corpus Linguistics. London: Continuum, 2009.

Beeby, Allison, Patricia Rodríguez-Inés e Pilar Sánchez-Gijón. "Introduction." Corpus Use and Translating. Eds. Allison Beeby, Patricia Rodríguez-Inés e Pilar Sánchez-Gijón. Amsterdam: John Benjamins Publishing Company, 2009. 1-8.

Bernardini, Silvia. Corpora and Language Learners. Studies in Corpus Linguistics. Amsterdam: John Benjamins Publishing Company, 2004.

Collocations in Translated Language: Combining Parallel, Comparable and Reference Corpora. Fourth Corpus Linguistics Conference. Birmingham: University of Birmingham, 2012.

Bernardini, Silvia, Federico Zanettin, Dominic Stewart (eds.). Corpora in Translator Education. Manchaster: St Jerome Publishing. 2003.

Berruto, Gaetano. La sociolinguistica. Bologna: Zanichelli, 1974.

Bolasco, Sergio. "Corpora e liste di frequenza d'uso: criteri e tecniche per l'analisi automatica dei testi." Lessico e apprendimenti. Eds. Monica Barni, Donatella Troncarelli e Carla Bagna. Milano: Franco Angeli, 2008. 113-42. 
Boulton, Alex. "Applying data-driven learning to the web." Multiple Affordances of Language Corpora for Data-driven Learning. Eds. Agnieszka Leńko-Szymańska e Alex Boulton. Amsterdam: John Benjamins Publishing Company, 2015. 267-295.

Bowker, Lynne. Computer-Aided Translation Technology: A Practical Introduction. University of Ottawa: Ottawa Press, Didactic of Translation Series, 2002.

"Corpus Resources for translators: academic luxury or professional necessity?" TradTerm 10, 2004: 213-247.

Bowker, Lynne e Jennifer Pearson. Working with Specialized Text: A Practical Guide to Using Corpora. UK: Routledge, 2002.

Bowker, Lynne e Michael Barlow. "A comparative evaluation of bilingual concordancers and translation memory systems." Topics for Language Resources in Translation and Localisation. Ed. Elia Yuste Rodrigo. Amsterdam: John Benjamins, 2008. 1-22.

Bruni, Francesco e Tommaso Raso (eds.). Manuale dell'italiano professionale. Bologna: Zanichelli, 2002.

Castagnoli, Sara. "Using the Web as a Source of LSP Corpora in the Terminology Classroom." Wacky! Working Papers on the Web as Corpus. Eds. Marco Baroni e Silvia Bernardini. Bologna: Gedit Edizioni, 2006. 159-72.

Chen, Meilin e Jane Flowerdew. "A critical review of research and practice in data-driven learning (DDL) in the academic writing classroom." International Journal of Corpus Linguistics 23.3 (2018): 335-69. Https://doi.org/10.1075/ijcl.16130.che. 22 Nov 2019.

Chiari, Isabella. "La chiave probabilistica delle lingue: teoria linguistica e applicazioni computazionali." Linguaggio, mente e società. Eds. Ludovico Fulci e Enrico Sciubba. Roma: Euroma La Goliardica, 2008. 55-79.

Cortelazzo Michele e Federica Pellegrino. Guida alla scrittura istituzionale. Bari: Laterza, 2003.

De Mauro, Tullio. La fabbrica delle parole. Torino: Utet Libreria, 2005.

Erdman-Thomsen, Hanne. Term Banks. The Encyclopedia of Applied Linguistics. Ed. Carol Chapelle. Oxford: Blackwell Publishing Ltd, 2012.

1-6. Https://doi.org/10.1002/9781405198431.wbeal1210. 13 Oct 2019.

Fantinuoli, Claudio e Federico Zanettin (eds.). New directions in corpusbased translation studies. Berlin: Language Science. 2015. 
Gavioli, Laura. Exploring Corpora for ESP Learning. Studies in Corpus Linguistics. Amsterdam: John Benjamins Publishing Company, 2005.

Gallego-Hernández, Daniel. "The use of corpora as translation resources: A study based on a survey of Spanish professional translators." Perspectives: Studies in Translatology 23.3 (2015): 375-391. Https://doi.org/10.1080/0907676X.2014.964269. 22 Nov 2019.

Hause, Juliane. "Translation and Bilingual Cognition." Language and Bilingual Cognition. Eds. Vivian Cook e Benedetta Bassetti. New York: Psychology Press, 2011: 519-528.

Hoey, Michael. Lexical Priming: A New Theory of Words and Language. Abingdon: Routledge, 2005.

Hüttner, Julia, Ute Smit e Barbara Mehlmauer-Larcher "ESP teacher education at the interface of theory and practice: Introducing a model of mediated corpus-based genre analysis." System 37.1 (2009): 99-109. Https://doi.org/10.1016/j.system.2008.06.003. 22 Nov 2019.

Johansson, Stig. Seeing through Multilingual Corpora: On the use of corpora in contrastive studies. Amsterdam: John Benjamins Publishing Company, 2007.

Kenny, Dorothy e Andy Way. "Teaching machine translation and translation technology: a contrastive study." The MT Summit VIII Workshop on Teaching Translation. 2001. Santiago de Compostella. 13-17.

Laviosa, Sara. Corpus-based Translation Studies: Theory, Findings, Applications. Amsterdam: Rodopi, 2002.

Louw, William Ernest. "Irony in the text or insincerity in the writer? The diagnostic potential of semantic prosodies." Text and Technology: In Honour of John Sinclair. Eds. Mona Baker, Gill Francis ed Elena Tognini-Bonelli. Amsterdam: John Benjamins, 1993. 157-176.

"Contextual Prosodic Theory: Bringing Semantic Prosodies to Life." Words in Context. In Honour of John Sinclair. Eds. Cris Heffer e Helen Sauntson. Birmingham: ELR, 2000. 48-94.

Malmkjær, Kirsten. "On a Pseudo-Subversive use of Corpora in Translation Training." Corpora in Translator Education. Eds. Federico Zanettin, Silvia Bernardini e Dominic Stewart. Manchester: St Jerome, 2003. 119-134.

McEnery, Tony, Richard Xiao e Yukio Tono. Corpus-Based Language Studies: an advanced resource book. Routledge, 2006. 
McEnery, Tony e Wilson, Andrew. Corpus Linguistics. Edinburgh: Edinburgh University Press, 1996.

Mauranen, Anna. "Strange Strings in Translated Language: A Study on Corpora." Intercultural Faultlines: Research Models in Translation Studies I. Ed. Maeve Olohan. London: Routledge. 2000, 119-142.

Olohan, Maeve. "Corpus Linguistics and Translation Studies: Interaction and Reaction." Linguistica Antverpiensia 1 (2002): 419-29.

-Introducing Corpora in Translation Studies. London: Routledge, 2004.

Olohan, Maeve and Mona Baker. "Reporting that in Translated English: Evidence for Subconscious Processes of Explicitation?" Across Languages and Cultures 1.2 (2000): 141-58.

Nigro, Maria Giovanna. Il linguaggio specialistico del turismo. Roma: Aracne, 2006.

Prandi, Michele. "Lessico naturale e lessici di specialità: tra descrizione e normalizzazione." Terminologia a colori. Eds. Franco Bertaccini, Sara Castagnoli e Francesca La Forgia. Bologna: Bononia, 2010. 53-84.

Riediger, Hellmut. "Tradurre col computer: Dalla traduzione automatica al CAT e ritorno." Fondazione Milano. Http://www.fondazionemilano.eu/blogpress/weaver/2018/06/ 12/tradurre-col-computer/ 15 Oct 2019.

Scarpa, Federica. La traduzione specializzata. Un approccio didattico professionale. Milano: Hoepli, 2008.

Serianni, Luca. Italiani scritti. Bologna: Il Mulino, 2007.

Sinclair, John. Corpus, Concordance, Collocation. OUP, 1991.

Sinclair, John. "Language and Computing, Past and Present." Evidencebased LSP. Translation, Text and terminology. Eds. Khurshid Ahmand e Margaret Rogers. Bern: Peter Lang, 2007. 21-51.

Soffritti, Marcello. "Termontografia e innovazione della terminologia plurilingue." Terminologia a colori. Eds. Franco Bertaccini, Sara Castagnoli e Francesca La Forgia. Bologna: Bononia UP, 2010: 31-51.

Stubbs, Michael. Words and Phrases: Corpus Studies in Lexical Semantics. Oxford: Blackwell, 2001.

Tiberii, Paola. Dizionario delle collocazioni: Le combinazioni delle parole in italiano. Bologna: Zanichelli 2018.

Tirkkonen-Condit, Sonja. "Unique items - Over- or Under-represented in Translated Language?" Translation Universals. Do they Exist?, Eds. Anne Mauranen e Pekka Kujamäki. Amsterdam: John Benjamins, 2004. 175-84. 
Toury, Gideon. Descriptive Translation Studies and Beyond. Amsterdam: Benjamins, 1995.

Varantola, Krista. "Translators and disposable corpora." Corpora in Translator Education. Eds. Federico Zanettin, Silvia Bernardini e Dominic Stewart. Manchester: St Jerome, 2003. 55-70.

Vermeer, Hans. A Skopos Theory of Translation: (Some Arguments For and Against). Heidelberg: TEXTconTEXT-Verlag, 1996.

Wilkinson, Michale. "Using a Specialized Corpus to Improve Translation Quality." Translation Journal 9.3 (2005).

Wills, Wolfram. Übersetzungswissenschaft. Probleme und Metoden. Stuttgart: Klett, 1977.

Zanettin, Federico. Translation-Driven Corpora: Corpus Resources for Descriptive and Applied Translation Studies. London: Routledge, 2012.

Zuccheri, Serena. "Terminologia e corpora a supporto della didattica della lingua cinese in ambito specialistico." Quaderni di Linguistica e Studi Orientali / Working Papers in Linguistics and Oriental Studies 2 (2016): 291-322.

\section{RAČUNARSKI PRISTUPI ZA NASTAVU PREVOĐENJA STRUČNIH JEZIKA}

Članak predlaže pitanje eksperimentalnog i inovativnog kursa za studente drugog stepena italijanistike na kojem se poučavaju novi pristupi prevođenju stručnih tekstova. Uzimajući u obzir principe, procese i pristupe predložene od strane brojnih lingvista (npr. Wills; Hause; Erdman et al.), na početku kursa je studentima predstavljena tekstualna analiza prevođenja nastavivši sa istraživanjem ponuđenih mogućnosti zahvaljujući novim tehnologijama kao što su alati podrške prevođenju (CAT - Computer-assisted translation) koji su predloženi od strane određenog broja korpusnih lingvista (npr. Laviosa; Gavioli; Boulton; Zanetin i Fantinuoli). Studentima je predstavljena upotreba CAT alata uz zadatak da prevedu jedan službeni dokument iz slovenačkog na italijanski jezik tokom čega su bili vođeni kroz sve faze pripreme i izvršenja prevoda. Naime, učesnici kursa su na početku morali da analiziraju predviđeni tekst o sticanju akademskih titula, nakon čega su im predstavljene različite opcije koje su omogućene zahvaljujući IT uređajima kao što su online rečnici, glosari, terminološke baze podataka i lingvistički korpusi koji mogu biti od pomoći pri istraživanju glavnih leksičkih resursa i kolokacijskih problema. S ciljem da se dodatno poboljša i ispita osnovna stručna terminologija predstavljeni su glavni principi ispunjavanja komparativnih korpusa 
manjeg obima uz softverske alate za istraživanje korpusa takvih dimenzija. Rezultati ovog naučnog istraživanja su upoređeni sa prevodilačkim rješenjima ponuđenim od strane alata za automatsko prevođenje, kao i sa prevodima predloženim od strane profesionalnih prevodilaca i maternjih govornika italijanskog jezika. Zaključci izvedeni iz eksperimentalnog kursa imaju implikacije za praktičnu nastavu i prevodilačka rješenja.

Ključne riječi: CAT alati, korpusi komparativnog jezika manjeg obima, pristupi u nastavi prevođenja 
APPENDICE

Tabella 1: Alcune soluzioni traduttive.

\begin{tabular}{|c|c|c|c|}
\hline Lingua & SLOVENO & ITALIANO & MemSource Cloud \\
\hline Campo & $\begin{array}{l}\text { Akademsko } \\
\text { področje }\end{array}$ & $\begin{array}{l}\text { Campo } \\
\text { accademico }\end{array}$ & \\
\hline Termine & Program & $\begin{array}{l}\text { Piano di studi } \\
\text { *e non } \\
\text { programma di } \\
\text { studi } \\
\end{array}$ & $\begin{array}{l}\text { Programma di } \\
\text { studi, } \\
\text { corso }\end{array}$ \\
\hline $\begin{array}{l}\text { Categoria } \\
\text { grammaticale }\end{array}$ & Samostalnik & Sostantivo & \\
\hline Definizione & $\begin{array}{l}\text { Skupek nalog, del, } \\
\text { ki se določijo za } \\
\text { uresničitev } \\
\text { študijskih ciljev }\end{array}$ & $\begin{array}{l}\text { Il programma } \\
\text { che lo studente } \\
\text { universitario è } \\
\text { tenuto a } \\
\text { redigere } \\
\text { all'inizio del } \\
\text { corso di laurea, } \\
\text { indicando le } \\
\text { materie e gli } \\
\text { esami che } \\
\text { intende } \\
\text { frequentare e } \\
\text { sostenere } \\
\end{array}$ & \\
\hline $\begin{array}{l}\text { Collocazione } \\
\text { con verbi }\end{array}$ & $\begin{array}{l}\text { Vpisati študijski } \\
\text { program, sprejeti, } \\
\text { določiti program, } \\
\text { uresničiti študijski } \\
\text { program } \\
\end{array}$ & $\begin{array}{l}\text { Definire, } \\
\text { prestabilire, } \\
\text { adottare un } \\
\text { piano di studi }\end{array}$ & $\begin{array}{l}\text { Essere iscritti a / } \\
\text { realizzare / offrire } \\
\text { un programma di } \\
\text { studo }\end{array}$ \\
\hline $\begin{array}{l}\text { Collocazioni } \\
\text { con aggettivi }\end{array}$ & $\begin{array}{l}\text { Doktorski, } \\
\text { magistrski, } \\
\text { končan, } \\
\text { visokošolski } \\
\text { (študijski program) }\end{array}$ & $\begin{array}{l}\text { Piano } \\
\text { formativo, di } \\
\text { ricerca, piano } \\
\text { scientifico- } \\
\text { formativo }\end{array}$ & \begin{tabular}{|l|} 
Dottorato, \\
programma di \\
master, \\
programma \\
d'istruzione di \\
terzo livello \\
\end{tabular} \\
\hline Frasi & $\begin{array}{l}\text { Končati/dokončati } \\
\text { študijski program }\end{array}$ & $\begin{array}{l}\text { Il piano di studi } \\
\text { deve esser } \\
\text { approvato }\end{array}$ & $\begin{array}{l}\text { Porta a un } \\
\text { diploma di } \\
\text { dottorato }\end{array}$ \\
\hline
\end{tabular}


2.

\begin{tabular}{|c|c|c|c|}
\hline Lingua & SLOVENO & ITALIANO & MemSource Cloud \\
\hline Campo & $\begin{array}{l}\text { Akademsko } \\
\text { področje }\end{array}$ & $\begin{array}{l}\text { Campo } \\
\text { accademico }\end{array}$ & \\
\hline Termine & Komisija & Commissione & Commissione \\
\hline $\begin{array}{l}\text { Categoria } \\
\text { grammaticale }\end{array}$ & Samostalnik & Sostantivo & \\
\hline Definizione & $\begin{array}{l}\text { Skupina ljudi } \\
\text { za opravljanje } \\
\text { posebnih } \\
\text { nalog, } \\
\text { izvoljena ali } \\
\text { imenovana } \\
\text { navadno za } \\
\text { določen čas } \\
\text { *nel SSKJ non } \\
\text { è presente } \\
\text { una } \\
\text { definizione } \\
\text { relativa alla } \\
\text { commissione } \\
\text { accademica }\end{array}$ & $\begin{array}{l}\text { Gruppo di } \\
\text { persone } \\
\text { investite di un } \\
\text { pubblico } \\
\text { incarico }\end{array}$ & \\
\hline $\begin{array}{l}\text { Collocazione } \\
\text { con verbi }\end{array}$ & $\begin{array}{l}\text { Koordinirati, } \\
\text { obravnavati, } \\
\text { povabiti, } \\
\text { imenovati } \\
\text { komisijo }\end{array}$ & $\begin{array}{l}\text { La } \\
\text { commissione } \\
\text { valuta, } \\
\text { delibera, } \\
\text { decade.. }\end{array}$ & $\begin{array}{l}\text { La commissione } \\
\text { vara, propone, } \\
\text { coordina, esamina }\end{array}$ \\
\hline $\begin{array}{l}\text { Collocazioni } \\
\text { con aggettivi }\end{array}$ & & $\begin{array}{l}\text { Commissione } \\
\text { giudicatrice, } \\
\text { esaminatrice }\end{array}$ & \\
\hline Frasi & $\begin{array}{l}\text { Komisija za } \\
\text { oceno, } \\
\text { komisija za } \\
\text { zagovor, člani } \\
\text { komisije za } \\
\text { odvzem } \\
\text { doktorata }\end{array}$ & $\begin{array}{l}\text { Commissione } \\
\text { giudicatrice } \\
\text { designata per } \\
\text { la valutazione }\end{array}$ & $\begin{array}{l}\text { Commissione per } \\
\text { la valutazione }\end{array}$ \\
\hline
\end{tabular}


3.

\begin{tabular}{|c|c|c|c|}
\hline Lingua & SLOVENO & ITALIANO & MemSource Cloud \\
\hline Campo & $\begin{array}{l}\text { Akademsko } \\
\text { področje }\end{array}$ & $\begin{array}{l}\text { Campo } \\
\text { accademico }\end{array}$ & \\
\hline Termine & Kandidat & Candidato & Candidato \\
\hline $\begin{array}{l}\text { Categoria } \\
\text { grammaticale }\end{array}$ & Samostalnik & Sostantivo & \\
\hline Definizione & $\begin{array}{l}\text { Kdor si } \\
\text { prizadeva ali se } \\
\text { poteguje za } \\
\text { naslov }\end{array}$ & $\begin{array}{l}\text { Chi si presenta } \\
\text { a un esame/ } \\
\text { concorso o } \\
\text { aspira ad una } \\
\text { carica }\end{array}$ & \\
\hline $\begin{array}{l}\text { Collocazione } \\
\text { con verbi }\end{array}$ & $\begin{array}{l}\text { Kandidat mora, } \\
\text { izpolnjuje, } \\
\text { predloži, prosi, } \\
\text { odda prijavo } \\
\text { teme }\end{array}$ & $\begin{array}{l}\text { Il candidato } \\
\text { collabora, } \\
\text { dimostra, } \\
\text { contribuisce, } \\
\text { svolge, } \\
\text { presenta }\end{array}$ & $\begin{array}{l}\text { Il candidato } \\
\text { soddisfa i requisiti } \\
\text { / le condizioni, } \\
\text { adempie ai criteri }\end{array}$ \\
\hline $\begin{array}{l}\text { Collocazioni } \\
\text { con aggettivi }\end{array}$ & $\begin{array}{l}\text { Določen, } \\
\text { izvoljen, } \\
\text { usposobljen } \\
\text { kandidat }\end{array}$ & $\begin{array}{l}\text { Candidato } \\
\text { meritevole, } \\
\text { singolo, } \\
\text { giovane }\end{array}$ & $\begin{array}{l}\text { Candidato eletto/ } \\
\text { considerato } \\
\text { idoneo a }\end{array}$ \\
\hline Frasi & $\begin{array}{l}\text { Kandidati } \\
\text { predispozicijo } \\
\text { predloži } \\
\text { komisiji, } \\
\text { kandidat } \\
\text { odgovori na } \\
\text { postavljena } \\
\text { vprašanja }\end{array}$ & $\begin{array}{l}\text { Il candidato } \\
\text { risulta } \\
\text { ammesso, la } \\
\text { commissione } \\
\text { ritiene che il } \\
\text { candidato sia } \\
\text { idoneo }\end{array}$ & $\begin{array}{l}\text { Presentate alla } \\
\text { commissione la } \\
\text { disposizione }\end{array}$ \\
\hline
\end{tabular}

4.

\begin{tabular}{|l|l|l|l|}
\hline Lingua & \multicolumn{1}{|c|}{ SLOVENO } & \multicolumn{1}{|c|}{ ITALIANO } & MemSource Cloud \\
\hline Campo & $\begin{array}{l}\text { Akademsko } \\
\text { področje }\end{array}$ & $\begin{array}{l}\text { Campo } \\
\text { accademico }\end{array}$ & \\
\hline Termine & Naziv & Titolo di studio & $\begin{array}{l}\text { Titolo di } \\
\text { formazione, } \\
\text { grado }\end{array}$ \\
\hline $\begin{array}{l}\text { Categoria } \\
\text { grammaticale }\end{array}$ & Samostalnik & Sostantivo & \\
\hline Definizione & Višja, visoka & Qualifica che & \\
\hline
\end{tabular}




\begin{tabular}{|c|c|c|c|}
\hline & $\begin{array}{l}\text { stopnja } \\
\text { izobrazbe, } \\
\text { naslov }\end{array}$ & $\begin{array}{l}\text { tocca di diritto } \\
\text { ad una persona } \\
\text { per il livello di } \\
\text { studi raggiunto }\end{array}$ & \\
\hline $\begin{array}{l}\text { Collocazione } \\
\text { con verbi }\end{array}$ & $\begin{array}{l}\text { Veljati, izvoliti, } \\
\text { prositi za naziv }\end{array}$ & $\begin{array}{l}\text { Conseguire, } \\
\text { rilasciare, } \\
\text { emettere, } \\
\text { possedere un } \\
\text { titolo di studio }\end{array}$ & $\begin{array}{l}\text { Conseguire } \\
\text { /possedere un } \\
\text { titolo di studio }\end{array}$ \\
\hline $\begin{array}{l}\text { Collocazioni } \\
\text { con aggettivi }\end{array}$ & $\begin{array}{l}\text { Visok, } \\
\text { ustrezen, } \\
\text { veljaven, } \\
\text { pridobljen, } \\
\text { akademski } \\
\text { naziv }\end{array}$ & $\begin{array}{l}\text { Titolo } \\
\text { accademico, } \\
\text { doppio, titolo } \\
\text { estero }\end{array}$ & $\begin{array}{l}\text { Promuovere al } \\
\text { grado di }\end{array}$ \\
\hline Frasi & $\begin{array}{l}\text { Izvolitve v } \\
\text { nazive } \\
\text { temeljijo na.., } \\
\text { naziv ugasne, } \\
\text { doba izvolitve } \\
\text { v naziv }\end{array}$ & $\begin{array}{l}\text { Il candidata ha } \\
\text { conseguito un } \\
\text { titolo di studi, } \\
\text { il titolo di } \\
\text { studio è stato } \\
\text { confermato } \\
\text { dalla } \\
\text { commissione }\end{array}$ & $\begin{array}{l}\text { Conferire il titolo } \\
\text { accademico di }\end{array}$ \\
\hline
\end{tabular}

5.

\begin{tabular}{|c|c|c|c|}
\hline Lingua & SLOVENO & ITALIANO & Memsource Cloud \\
\hline Campo & $\begin{array}{l}\text { Akademsko } \\
\text { področje }\end{array}$ & $\begin{array}{l}\text { Campo } \\
\text { accademico }\end{array}$ & \\
\hline Termine & Disertacija & Tesi & Tesi di dottorato \\
\hline $\begin{array}{l}\text { Categoria } \\
\text { grammaticale }\end{array}$ & Samostalnik & Sostantivo & \\
\hline Definizione & $\begin{array}{l}\text { Znanstvena } \\
\text { razprava za } \\
\text { dosego } \\
\text { doktorskega } \\
\text { naslova }\end{array}$ & $\begin{array}{l}\text { Dissertazione } \\
\text { scritta che un } \\
\text { candidato } \\
\text { presenta e } \\
\text { discute davanti a } \\
\text { una commissione } \\
\text { di docenti alla } \\
\text { fine di un corso } \\
\text { di laurea } \\
\text { universitario }\end{array}$ & \\
\hline
\end{tabular}




\begin{tabular}{|l|l|l|l|}
\hline $\begin{array}{l}\text { Collocazione } \\
\text { con verbi }\end{array}$ & $\begin{array}{l}\text { Zagovarjati, } \\
\text { oddati, } \\
\text { prijaviti, } \\
\text { predlagati } \\
\text { disertacijo }\end{array}$ & $\begin{array}{l}\text { Discutere, } \\
\text { presentare, } \\
\text { scrivere una tesi }\end{array}$ & $\begin{array}{l}\text { Redazione della } \\
\text { tesi, } \\
\text { Completare / } \\
\text { preparare la tesi di } \\
\text { dottorato }\end{array}$ \\
\hline $\begin{array}{l}\text { Collocazioni } \\
\text { con aggettivi }\end{array}$ & $\begin{array}{l}\text { Doktorska } \\
\text { disertacija }\end{array}$ & Tesi magistrale & Tesi di dottorato \\
\hline Frasi & $\begin{array}{l}\text { Komisija za } \\
\text { zagovor } \\
\text { disertacije, } \\
\text { primernost } \\
\text { teme in } \\
\text { dispozicije } \\
\text { disertacije }\end{array}$ & $\begin{array}{l}\text { Il candidato ha } \\
\text { discusso la tesi, } \\
\text { non vanno } \\
\text { consegnate le } \\
\text { tesi in formato } \\
\text { cartaceo } \\
\text { Tesi di dottorato, } \\
\text { di laurea }\end{array}$ & $\begin{array}{l}\text { Commissione di } \\
\text { difesa, } \\
\text { Discussione della } \\
\text { tesi }\end{array}$ \\
& \multicolumn{2}{|l}{} \\
\hline
\end{tabular}

6.

\begin{tabular}{|l|l|l|l|}
\hline Lingua & \multicolumn{1}{|c|}{ SLOVENO } & \multicolumn{1}{|c|}{ ITALIANO } & MemSource Cloud \\
\hline Campo & $\begin{array}{l}\text { Akademsko } \\
\text { področje }\end{array}$ & $\begin{array}{l}\text { Campo } \\
\text { accademico }\end{array}$ & \\
\hline Termine & Ocena & $\begin{array}{l}\text { Valutazione } \\
\text { *non voto }\end{array}$ & valutazione \\
\hline $\begin{array}{l}\text { Categoria } \\
\text { grammatical } \\
\text { e }\end{array}$ & Samostalnik & Sostantivo & \\
\hline Definizione & $\begin{array}{l}\text { Ovrednotenje } \\
\text { znanja v šoli }\end{array}$ & $\begin{array}{l}\text { Nel campo } \\
\text { accademico, } \\
\text { documento } \\
\text { su cui viene } \\
\text { espresso il } \\
\text { giudizio in } \\
\text { merito ai } \\
\text { risultati } \\
\text { aggiunti }\end{array}$ & \\
\hline $\begin{array}{l}\text { Collocazione } \\
\text { con verbi }\end{array}$ & $\begin{array}{l}\text { Navesti oceno, } \\
\text { zavrniti oceno }\end{array}$ & $\begin{array}{l}\text { Effettuare, } \\
\text { formulare, } \\
\text { assegnare } \\
\text { una } \\
\text { valutazione }\end{array}$ & $\begin{array}{l}\text { Indicare / rifiutare la } \\
\text { valutazione }\end{array}$ \\
\hline $\begin{array}{l}\text { Collocazioni } \\
\text { con aggettivi }\end{array}$ & $\begin{array}{l}\text { Povprečna, } \\
\text { negativna, }\end{array}$ & $\begin{array}{l}\text { Valutazione } \\
\text { individuale, }\end{array}$ & $\begin{array}{l}\text { Punteggio medio } \\
\text { complessivo, }\end{array}$ \\
\hline
\end{tabular}




\begin{tabular}{|l|l|l|l|}
\hline & pozitivna & $\begin{array}{l}\text { esterna, } \\
\text { finale }\end{array}$ & $\begin{array}{l}\text { valutazione / esito } \\
\text { positivo/negativo }\end{array}$ \\
\hline Frasi & $\begin{array}{l}\text { Komisija za } \\
\text { oceno, } \\
\text { poročevalec za } \\
\text { oceno } \\
\text { kandidatove } \\
\text { usposobljenos } \\
\text { ti }\end{array}$ & $\begin{array}{l}\text { La } \\
\text { commission } \\
\text { e ha } \\
\text { formulato } \\
\text { una } \\
\text { valutazione } \\
\text { complessiva } \\
\text { valutazione, } \\
\text { Relazione/presentazion } \\
\text { e della commissione }\end{array}$ & \\
& $\begin{array}{l}\text { la } \\
\text { referente } \\
\text { per la } \\
\text { valutazione } \\
\text { è la prof... }\end{array}$ & \\
& &
\end{tabular}

7.

\begin{tabular}{|l|l|l|l|}
\hline Lingua & \multicolumn{1}{|c|}{ SLOVENO } & \multicolumn{1}{|c|}{ ITALIANO } & MemSource Cloud \\
\hline Campo & $\begin{array}{l}\text { Akademsko } \\
\text { področje }\end{array}$ & $\begin{array}{l}\text { Campo } \\
\text { accademico }\end{array}$ & \\
\hline Termine & Zagovor & $\begin{array}{l}\text { Discussione } \\
\text { della tesi } \text { *e } \\
\text { non difesa della } \\
\text { tesi }\end{array}$ & presentazione \\
\hline $\begin{array}{l}\text { Categoria } \\
\text { grammaticale }\end{array}$ & Samostalnik & Frase nominale & \\
\hline Definizione & $\begin{array}{l}\text { Zagovor dela, } \\
\text { naloge pred } \\
\text { komisijo }\end{array}$ & $\begin{array}{l}\text { esame finale } \\
\text { del corso di } \\
\text { studi } \\
\text { universitari, in } \\
\text { cui il laureando } \\
\text { illustra il } \\
\text { proprio lavoro }\end{array}$ & \\
\hline $\begin{array}{l}\text { Collocazione } \\
\text { con verbi }\end{array}$ & $\begin{array}{l}\text { Zaključiti, } \\
\text { podpisati, } \\
\text { urejati, } \\
\text { zavrniti, } \\
\text { opraviti } \\
\text { zagovor }\end{array}$ & $\begin{array}{l}\text { Svolgere, } \\
\text { tenere, } \\
\text { sostenere la } \\
\text { discussione } \\
\text { della tesi }\end{array}$ & $\begin{array}{l}\text { Fare, completare, } \\
\text { rifiutare il } \\
\text { dottorato }\end{array}$ \\
\hline
\end{tabular}




\begin{tabular}{|l|l|l|l|}
\hline $\begin{array}{l}\text { Collocazioni } \\
\text { con aggettivi }\end{array}$ & Javni zagovor & $\begin{array}{l}\text { Discussione } \\
\text { pubblica, } \\
\text { privata, } \\
\text { generale }\end{array}$ & Difesa pubblica \\
\hline Frasi & $\begin{array}{l}\text { Komisija za } \\
\text { zagovor } \\
\text { disertacije, } \\
\text { zagovor } \\
\text { fakulteta } \\
\text { objavi }\end{array}$ & $\begin{array}{l}\text { La discussione } \\
\text { della tesi si } \\
\text { svolge di fronte } \\
\text { a una } \\
\text { commissione } \\
\text { paritetica }\end{array}$ & $\begin{array}{l}\text { Commissione per } \\
\text { la difesa del } \\
\text { dottorato }\end{array}$ \\
\hline
\end{tabular}

8.

\begin{tabular}{|c|c|c|c|}
\hline Lingua & SLOVENO & ITALIANO & $\begin{array}{l}\text { MemSource } \\
\text { Cloud }\end{array}$ \\
\hline Campo & $\begin{array}{l}\text { Akademsko } \\
\text { področje }\end{array}$ & $\begin{array}{l}\text { Campo } \\
\text { accademico }\end{array}$ & \\
\hline Termine & Diploma & Diploma & Diploma \\
\hline $\begin{array}{l}\text { Categoria } \\
\text { grammaticale }\end{array}$ & Samostalnik & Sostantivo & \\
\hline Definizione & $\begin{array}{l}\text { Dokument o } \\
\text { uspešni } \\
\text { dovršitvi } \\
\text { šolanja, na višji } \\
\text { ali visoki šoli }\end{array}$ & $\begin{array}{l}\text { Documento che } \\
\text { attesta il } \\
\text { conseguimento } \\
\text { di un titolo di } \\
\text { studio }\end{array}$ & \\
\hline $\begin{array}{l}\text { Collocazione } \\
\text { con verbi }\end{array}$ & $\begin{array}{l}\text { Podeliti, } \\
\text { pridobiti, imeti } \\
\text { diplomo }\end{array}$ & $\begin{array}{l}\text { Conseguire, } \\
\text { sottoscrivere il } \\
\text { diploma }\end{array}$ & $\begin{array}{l}\text { Rilasciare / } \\
\text { ottenere / } \\
\text { riconoscere il } \\
\text { diploma }\end{array}$ \\
\hline $\begin{array}{l}\text { Collocazioni } \\
\text { con aggettivi }\end{array}$ & $\begin{array}{l}\text { Specialistična, } \\
\text { magistrska, } \\
\text { izvirna diploma }\end{array}$ & $\begin{array}{l}\text { Diploma doppio, } \\
\text { unico, finale }\end{array}$ & $\begin{array}{l}\text { Corso di } \\
\text { diploma o di } \\
\text { laurea } \\
\text { magistrale }\end{array}$ \\
\hline Frasi & $\begin{array}{l}\text { Diplomanti si } \\
\text { pridobijo } \\
\text { diplomo, } \\
\text { komisija } \\
\text { prekliče } \\
\text { veljavnost } \\
\text { izdane diplome }\end{array}$ & $\begin{array}{l}\text { Ha conseguito il } \\
\text { diploma di } \\
\text { specializzazione } \\
\text { presso l'ateneo }\end{array}$ & $\begin{array}{l}\text { Titolare del } \\
\text { diploma, } \\
\text { Revocare il } \\
\text { diploma }\end{array}$ \\
\hline
\end{tabular}


9.

\begin{tabular}{|c|c|c|c|}
\hline Lingua & SLOVENO & ITALIANO & $\begin{array}{l}\text { MemSource } \\
\text { Cloud }\end{array}$ \\
\hline Campo & $\begin{array}{l}\text { Akademsko } \\
\text { področje }\end{array}$ & $\begin{array}{l}\text { Campo } \\
\text { accademico }\end{array}$ & \\
\hline Termine & Senat & Senato & Senato \\
\hline $\begin{array}{l}\text { Categoria } \\
\text { grammaticale }\end{array}$ & Samostalnik & Sostantivo & \\
\hline Definizione & $\begin{array}{l}\text { najvišji } \\
\text { akademski in } \\
\text { strokovni } \\
\text { organ } \\
\text { univerze, ki ga } \\
\text { sestavljajo } \\
\text { visokošolski } \\
\text { učitelji }\end{array}$ & $\begin{array}{l}\text { Organo collegiale, } \\
\text { deputato al } \\
\text { governo delle } \\
\text { università }\end{array}$ & \\
\hline $\begin{array}{l}\text { Collocazione } \\
\text { con verbi }\end{array}$ & $\begin{array}{l}\text { Izvoliti, } \\
\text { sprejeti, } \\
\text { odločati, } \\
\text { odpovedati }\end{array}$ & $\begin{array}{l}\text { Il senato assume } \\
\text { (delle decisioni }\end{array}$ & $\begin{array}{l}\text { Eleggere; } \\
\text { adottare, } \\
\text { prende } \\
\text { decisione }\end{array}$ \\
\hline $\begin{array}{l}\text { Collocazioni } \\
\text { con aggettivi }\end{array}$ & $\begin{array}{l}\text { Senat članice, } \\
\text { univerze, } \\
\text { fakultete }\end{array}$ & $\begin{array}{l}\text { Senato } \\
\text { accademico }\end{array}$ & $\begin{array}{l}\text { Senato } \\
\text { dell'università }\end{array}$ \\
\hline Frasi & $\begin{array}{l}\text { Senat sprejme } \\
\text { zagovor } \\
\text { doktorata, } \\
\text { uvrstiti na } \\
\text { sejo senata }\end{array}$ & $\begin{array}{l}\text { Il senato } \\
\text { accademico è } \\
\text { stato sentito } \\
\text { secondo i } \\
\text { parametri stabiliti } \\
\text { dal consiglio di } \\
\text { amministrazione }\end{array}$ & $\begin{array}{l}\text { Il senato ha } \\
\text { adottato } \\
\text { /approvato }\end{array}$ \\
\hline
\end{tabular}

10.

\begin{tabular}{|l|l|l|l|}
\hline Lingua & \multicolumn{1}{|c|}{ SLOVENO } & \multicolumn{1}{|c|}{ ITALIANO } & $\begin{array}{c}\text { MemSource } \\
\text { Cloud }\end{array}$ \\
\hline Campo & $\begin{array}{l}\text { Akademsko } \\
\text { področje }\end{array}$ & $\begin{array}{l}\text { Campo } \\
\text { accademico }\end{array}$ & \\
\hline Termine & Doktorat & Dottorato & Dottorato \\
\hline $\begin{array}{l}\text { Categoria } \\
\text { grammaticale }\end{array}$ & Samostalnik & Sostantivo & \\
\hline
\end{tabular}




\begin{tabular}{|l|l|l|l|}
\hline Definizione & $\begin{array}{l}\text { Delo, izpit za } \\
\text { dosego } \\
\text { doktorskega } \\
\text { naslova }\end{array}$ & $\begin{array}{l}\text { Grado, titolo di } \\
\text { dottore } \\
\text { conseguito con } \\
\text { la laurea }\end{array}$ & \\
\hline $\begin{array}{l}\text { Collocazione } \\
\text { con verbi }\end{array}$ & $\begin{array}{l}\text { Odvzem, } \\
\text { odobritev, } \\
\text { zavrnitev } \\
\text { doktorata }\end{array}$ & $\begin{array}{l}\text { Conseguire, } \\
\text { pubblicare, }\end{array}$ & Ottenere \\
\hline $\begin{array}{l}\text { Collocazioni } \\
\text { con aggettivi }\end{array}$ & $\begin{array}{l}\text { Znanstveni, } \\
\text { umetnostni } \\
\text { doktorat }\end{array}$ & $\begin{array}{l}\text { Dottorato } \\
\text { bolognese } \\
\text { (relativo al } \\
\text { sistema di } \\
\text { Bologna), } \\
\text { innovativo.. }\end{array}$ & $\begin{array}{l}\text { Dottorato } \\
\text { scientifico/ } \\
\text { artistico }\end{array}$ \\
\hline Frasi & $\begin{array}{l}\text { Asistent z } \\
\text { doktoratom je } \\
\text { lahko.., } \\
\text { postopek za } \\
\text { pridobitev } \\
\text { doktorata }\end{array}$ & $\begin{array}{l}\text { La tesi di } \\
\text { dottorato deve } \\
\text { venir } \\
\text { pubblicata, } \\
\text { Crescente } \\
\text { capacità di } \\
\text { attrazione dei } \\
\text { dottorati } \\
\text { bolognesi } \\
\text { adottorato di } \\
\text { ricerca }\end{array}$ & $\begin{array}{l}\text { Procedura di } \\
\text { autorizzazione / } \\
\text { per ottenere il } \\
\text { dottorato }\end{array}$ \\
\hline
\end{tabular}

Tabella 2: Strumenti e risorse elettroniche on-line utilizzate in classe

\begin{tabular}{|ll|}
\hline \multicolumn{2}{|c|}{ Dizionari e vocabolari informatizzati monolingui } \\
\hline Accademia della Crusca & http://www.accademiadellacrusca.it/lingue \\
& speciali.shtml \\
Dizionario della lingua & https://dizionari.corriere.it/dizionario_italia \\
italiana (Sabatini & no/R/riconducibile.shtml \\
Coletti) & http://www.oxfordparavia.it \\
$\begin{array}{l}\text { Dizionario Oxford } \\
\text { Paravia On line }\end{array}$ & \\
$\begin{array}{l}\text { La Sitoteca - Biblioteca } \\
\text { delle opere di } \\
\text { consultazione e degli } \\
\text { strumenti per la ricerca } \\
\text { terminologica e }\end{array}$ \\
\hline
\end{tabular}




\begin{tabular}{ll}
\hline lessicale in Internet & \\
Macmillan Dictionary & http://www.macmillandictionary.com \\
On line & \\
Terminology Collection: & http://www.uva.fi/en/sites/terminology \\
Online Dictionaries & \\
Term-minator, dizionari & http://www.term-minator.eu/ita_diz.html; \\
ed enciclopedie, & http://www.term- \\
terminologia e glossari & minator.eu/ita_glossa.html \\
Vocabolario Treccani & http://www.treccani.it/vocabolario/vocabol \\
On line & $\underline{\text { ario/ }}$ \\
Yourdictionary & http://www.yourdictionary.com \\
\hline
\end{tabular}

\section{Dizionari informatizzati bilingui (basati su memorie di traduzione)}

\begin{tabular}{|c|c|}
\hline Eurocorpus & $\begin{array}{l}\text { https://evroterm.vlada.si/evrokorpus/index } \\
\text {.php?jezik=it }\end{array}$ \\
\hline Glosbe & https://sl.glosbe.com/en/sl \\
\hline Glossari.it & http://www.glossari.it \\
\hline Glossopedia & $\begin{array}{l}\text { http://www.glossapedia.com/wordpress/m } \\
\text { odelli-di-glossario }\end{array}$ \\
\hline Lexicool & http://www.lexicool.com \\
\hline Linguee & https://sl.linguee.com/ \\
\hline \multicolumn{2}{|c|}{ Ricerca automatica di collocazioni } \\
\hline $\begin{array}{l}\text { itSkELL: Italian corpus } \\
\text { for SkELL }\end{array}$ & $\begin{array}{l}\text { https://www.sketchengine.eu/itskell- } \\
\text { italian-corpus/ }\end{array}$ \\
\hline \multicolumn{2}{|c|}{ Banche dati terminologiche } \\
\hline IATE & https://iate.europa.eu/search/standard \\
\hline \multicolumn{2}{|r|}{ Corpora Online } \\
\hline $\begin{array}{l}\text { Corpus e Lessico di } \\
\text { Frequenza dell'Italiano } \\
\text { Scritto (CoLFIS) }\end{array}$ & http://linguistica.sns.it/CoLFIS/Home.htm \\
\hline Coris/codis & http://corpora.dslo.unibo.it/coris ita.html \\
\hline Perugia Corpus & $\begin{array}{l}\text { https://www.unistrapg.it/perugiacorpus/in } \\
\text { dex.html }\end{array}$ \\
\hline $\begin{array}{l}\text { GigaFida (Corpus } \\
\text { sloveno) }\end{array}$ & http://www.gigafida.net/ \\
\hline \multicolumn{2}{|c|}{ Strumenti per l'analisi testuale } \\
\hline Sketch Engine Suite & https://www.sketchengine.eu \\
\hline AntConc & $\begin{array}{l}\text { http://www.laurenceanthony.net/software. } \\
\text { html }\end{array}$ \\
\hline \multicolumn{2}{|c|}{ Piattaforma per la traduzione automatica } \\
\hline MemSource & https://www.memsource.com/ \\
\hline
\end{tabular}


Tabella 3: Creazione e denominazione dei file scaricati dal web

\begin{tabular}{|c|c|c|c|c|c|}
\hline $\begin{array}{l}\text { Nome } \\
\text { del file }\end{array}$ & Provenienza IT & $\begin{array}{l}\text { No } \\
\text { di } \\
\text { par } \\
\text { ole }\end{array}$ & $\begin{array}{l}\text { Nome } \\
\text { del file }\end{array}$ & Provenienza SL & $\begin{array}{l}\text { No di } \\
\text { parole }\end{array}$ \\
\hline $\begin{array}{l}\text { Dot1.it. } \\
\text { doc }\end{array}$ & $\begin{array}{l}\text { ITA - BO } \\
\text { Regolamento in } \\
\text { materia di Corsi di } \\
\text { dottorato } \\
\text { http://www.norm } \\
\text { ateneo.unibo.it/r } \\
\text { egolamento-in- } \\
\text { materia-di-corsi- } \\
\text { di-dottorato }\end{array}$ & 685 & $\begin{array}{l}\text { Dot1.sl. } \\
\text { doc }\end{array}$ & $\begin{array}{l}\text { SLO - KP Pravilnik } \\
\text { o zagovoru } \\
\text { doktorske } \\
\text { disertacije } \\
\text { http://www.fhs.u } \\
\text { pr.si/sl/resources } \\
\text { /files/o- } \\
\text { fakulteti/interni- } \\
\text { akti/pravilnik-o- } \\
\text { pipravi-in- } \\
\text { zagovoru- } \\
\text { doktoske- } \\
\text { disertacije181020 } \\
\text { 14.pdf }\end{array}$ & 6025 \\
\hline $\begin{array}{l}\text { Dot2.it. } \\
\text { doc }\end{array}$ & $\begin{array}{l}\text { ITA TS - } \\
\text { Regolamento sul } \\
\text { dottorato di } \\
\text { ricerca UNI TS } \\
\text { https://web.units. } \\
\text { it/sites/default/fil } \\
\text { es/nrm/allegati/N } \\
\text { uovo\%20Reg.\%20 } \\
\text { dottorato\%20di\% } \\
\text { 20ricerca\%20vige } \\
\text { nte\%20ott\%2020 } \\
\text { 15_1.pdf }\end{array}$ & 426 & $\begin{array}{l}\text { Dot2.sl. } \\
\text { doc }\end{array}$ & $\begin{array}{l}\text { SLO - L Pravilnik } \\
\text { o doktorskem } \\
\text { študiju UL } \\
\text { https://www.uni- } \\
\text { lj.si/studij/doktor } \\
\text { ski/pravila/ }\end{array}$ & 4916 \\
\hline $\begin{array}{l}\text { Dot3.it. } \\
\text { doc }\end{array}$ & $\begin{array}{l}\text { ITA RO DR } \\
\text { regolamento_mo } \\
\text { dificato } \\
\text { https://www.unir } \\
\text { oma1.it/sites/def } \\
\text { ault/files/regolam } \\
\text { enti/DR_regolam } \\
\text { ento_modificato. } \\
\text { pdf }\end{array}$ & $\begin{array}{l}805 \\
5\end{array}$ & $\begin{array}{l}\text { Dot3.sl. } \\
\text { doc }\end{array}$ & $\begin{array}{l}\text { SLO - MB } \\
\text { Pravilnik_o_dokto } \\
\text { rskem_študiju } \\
\text { https://www.fnm } \\
\text {.um.si/wp- } \\
\text { content/uploads/ } \\
\text { 2018/05/Pravilnik } \\
\text {-o-doktorskem- } \\
\text { studiju-na- } \\
\text { Univerzi-v- } \\
\text { Mariboru-012- } \\
\text { 2018-1.pdf }\end{array}$ & 17369 \\
\hline
\end{tabular}




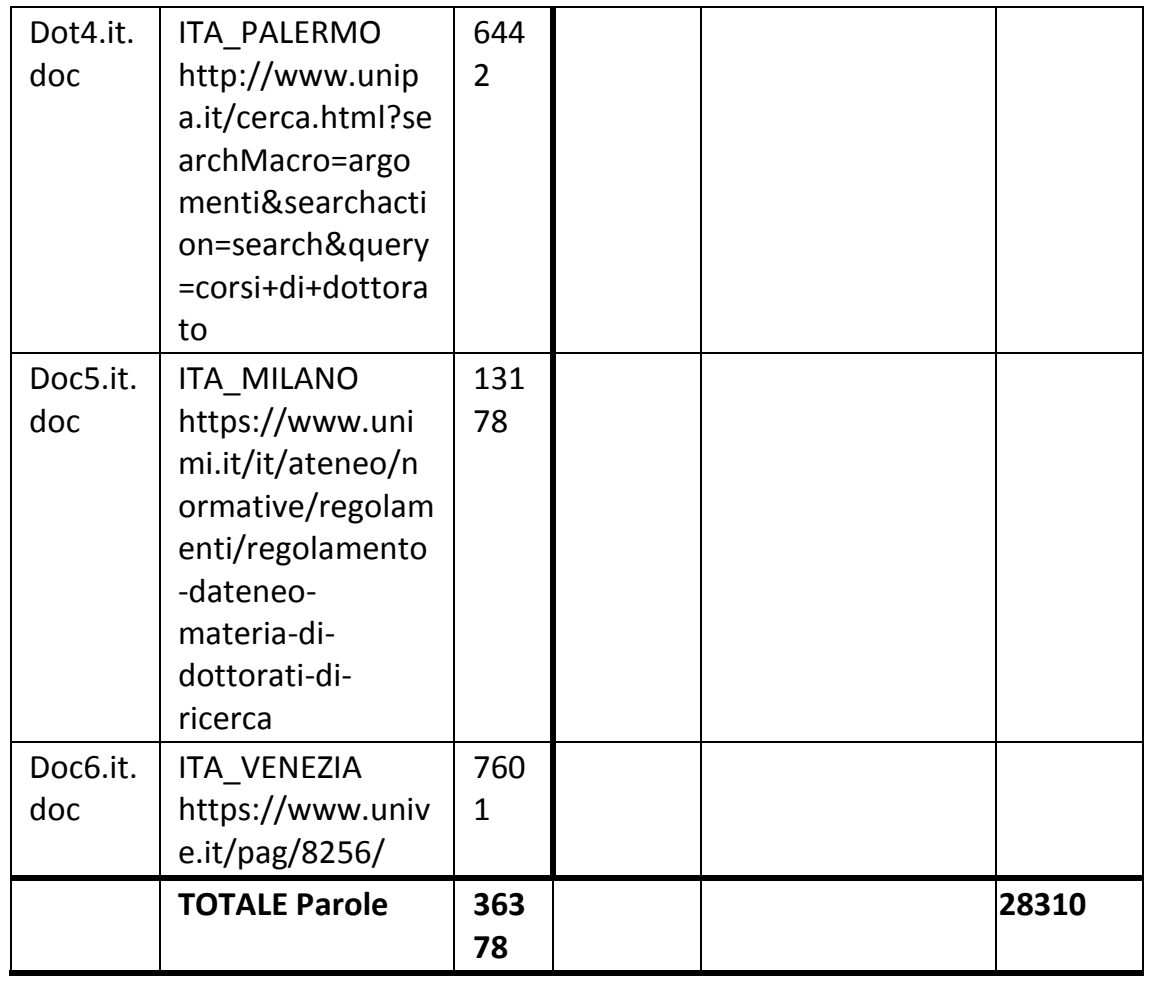

